\title{
The impact of vacancies on the stability of cubic phases in Sb-Te binary compounds
}

\author{
Xin Chen ${ }^{1,2,3}$, Xianqiang Liu', Yan Cheng ${ }^{1,4}$ and Zhitang Song
}

\begin{abstract}
Data retention ability and number of cycles are key properties of phase change materials in applications. Combining in situ heating transmission electron microscopy with ab initial calculations, we investigated the phase transitions of binary Sb-Te compounds. The calculations indicated that the vacancies in Te sites destroyed the framework of the cubic phase, which agrees well with the absence of cubic phases observed during in situ heating experiments when the Sb concentration exceeded 50\%. In contrast, the vacancies in Sb sites stabilized the cubic structure. Further analysis of the charge density maps revealed that the distribution of antibonding electrons may be the origin of the driving force for structural transitions. Furthermore, our results also showed that reducing the vacancies greatly increased the phase transition temperatures of both the amorphous-cubic and cubic-trigonal phases and therefore may improve the data retention ability and cyclability of phase change materials. This result also implies that doping Sb-Te compounds may provide an approach to discover novel phase change materials by reducing the amount of vacancies.
\end{abstract}

\section{Introduction}

Recent major breakthroughs that addressed the limitations ${ }^{1}$ of phase change memory $(\mathrm{PCM})$ are renewing interest in it as a promising next-generation technology for electronic data storage and computation ${ }^{2,3}$. If the performance of PCM could compete with that of dynamic random access memory (DRAM), completely new computer architectures would be enabled ${ }^{4}$. To date, the most widely studied systems lie on, or are in the vicinity of, a pseudobinary line that joins the stoichiometric compounds $\mathrm{Sb}_{2} \mathrm{Te}_{3}$ and GeTe. Among these compounds, the alloy $\mathrm{Ge}_{2} \mathrm{Sb}_{2} \mathrm{Te}_{5}$ has the best combination of properties: fast crystallization, amorphous phase stability, high endurance limit and excellent contrast; ${ }^{5}$ therefore, it has attracted the largest amount of attention. The crystalline phase utilized in GeSbTe-based PCMs that are currently

Correspondence: Xianqiang Liu (xqliu@bjut.edu.cn) or

Zhitang Song (ztsong@mail.sim.ac.cn)

${ }^{1}$ State Key Laboratory of Functional Materials for Informatics, Shanghai Institute of Micro-system and Information Technology, Chinese Academy of Sciences, 200050 Shanghai, China

${ }^{2}$ Institute of Microstructure and Property of Advanced Materials, Beijing

University of Technology, 100124 Beijing, China

Full list of author information is available at the end of the article. in use is the cubic metastable phase, which contains large quantities of vacancies ${ }^{6-8}$. The vacancies in the $\mathrm{Ge} / \mathrm{Sb}$ sublattice are intrinsic ${ }^{9}$ and have been demonstrated to play an important role in phase transitions and related properties $^{10-17}$.

Replacing DRAM with PCM is a formidable challenge because of the combined requirements of a fast switching speed and extremely high cycle numbers. Although a Sbrich GeSbTe PCM demonstrated $10^{11}$ cycles under accelerated testing conditions ${ }^{18}$, the required cycle numbers for a DRAM replacement have not been achieved ${ }^{4}$. One of the main failure mechanisms of PCM devices is void formation over the bottom electrode contact. The other main failure mechanism is elemental segregation, which leads to poor data retention when the cell can no longer be switched to the high-resistance state or does not remain in the high-resistance state since Sb-rich alloys have low crystallization temperatures ${ }^{4}$.

In terms of the failure mechanism in GeSbTe-based PCMs, Sb-Te alloys have advantages compared to GeSbTe alloys, e.g., a simple composition, which prevents the phase separation that occurs in GeSbTe alloys. As the parent material of GeSbTe $\mathrm{PCMs}, \mathrm{Sb}_{2} \mathrm{Te}_{3}$ and its promise 
have been ignored for a substantial amount of time. Recently, Zheng et al. proved that the metastable facecentered cubic (FCC) $\mathrm{Sb}_{2} \mathrm{Te}_{3}$ phase does exist ${ }^{8}$. A series of studies showed that-doped $\mathrm{Sb}_{2} \mathrm{Te}_{3}$ exhibits excellent properties that are required for $\mathrm{PCMs}^{19,20}$, such as a fast phase transition speed, good reversibility at elevated temperatures and good thermal stability at room temperature. In 2017, Rao et al. demonstrated that the $\mathrm{Sc}_{0.2} \mathrm{Sb}_{2} \mathrm{Te}_{3}$ compound that they designed achieved a writing speed of only 700 picoseconds ${ }^{7}$. The ultrafast crystallization is due to the reduced stochasticity of nucleation through geometrically matched and robust scandium telluride (ScTe) chemical bonds that stabilize the crystal precursors in the amorphous state. This makes $\mathrm{Sb}_{2} \mathrm{Te}_{3}$ an attractive competitor to DRAM because of the ultrafast writing speed. Unfortunately, as a DRAM replacement, $\mathrm{Sb}_{2} \mathrm{Te}_{3}$ possesses the same shortcomings as GeSbTe. Due to the large quantities of volatile vacancies in the metastable phase, the cubic phase has a strong tendency to transform into a stable trigonal phase during cyclic writing processes. The vacancy ordering and evaporation from the cubic phase to the trigonal phase result in a density change ${ }^{21}$, which causes void formation, triggering the failure of $\mathrm{PCMs}^{4}$. Additionally, pure $\mathrm{Sb}_{2} \mathrm{Te}_{3}$ has a very low crystallization temperature and thus a poor data retention ability.

Here, in this work, we systematically investigated the phase transitions of $\mathrm{Sb}-\mathrm{Te}$ binary compounds between $\mathrm{Sb}_{2} \mathrm{Te}_{3}$ and $\mathrm{Sb}_{2} \mathrm{Te}$ by combining in situ heating transmission electron microscopy (TEM) and ab initio calculations. The in situ TEM experiments showed that vacancies play a central role in the phase transition temperatures of $\mathrm{Sb}-\mathrm{Te}$ compounds. The ab initio calculations revealed that the vacancy type is the factor that determines the existence of a cubic phase and that the amount of vacancies has a great impact on the phase transition temperatures.

\section{Materials and methods}

\section{Film preparation and characterization}

The TEM samples $(\sim 15 \mathrm{~nm})$ were deposited on copper grids with an ultrathin carbon support by varying the RF sputtering power on $\mathrm{Sb}$ and $\mathrm{Sb}_{2} \mathrm{Te}_{3}$ targets by physical vapor deposition (PVD). The deposition rate was measured from cross-section samples by scanning electron microscopy (SEM). The sample composition was tested by energy dispersive spectroscopy (EDS) (shown in Supplementary Fig. 10). The in situ heating experiments were performed with TEM (JEOL 2100F) with a heating rate of $10^{\circ} \mathrm{C} / \mathrm{min}$ using a single tilt holder (Gatan 628 ). To alleviate the influence of electron irradiation, the samples were exposed to the beam for a short time at the capturing temperature, and the area was changed for each observation. High-resolution TEM (HRTEM) experiments were performed on a JEOL-ARM300F instrument with a double Cs corrector operating at $300 \mathrm{kV}$.

\section{$\mathrm{Ab}$ initio theoretical simulation}

The ab initio calculations were carried out by employing the Vienna ab initio simulation package $\left(\right.$ VASP) ${ }^{22}$ with projected augmented wave (PAW) pseudopotentials ${ }^{23}$ and Perdew-Burke-Ernzerhof (PBE) functionals ${ }^{24}$. The $14.942 \times 14.942 \times 31.697(\AA)$ supercell of the FCC structure was built. For the stable structures with a trigonal phase with van der Waals (vdW) gaps, the DFT-D2 method of the Grimme scheme ${ }^{25}$ was used for corrections. The energy cut-off was set to $235 \mathrm{eV}$, and the $\Gamma$ point was used in the Brillouin zone sum. Chemical bonding analysis was carried out by combining a charge density map with the COHP method ${ }^{26}$. The Local Orbital Basis Suite Towards Electronic-Structure Reconstruction (LOBSTER) code $^{27}$ handles the DFT calculation results and projects them onto localized atomic basis sets $^{28}$. The activation energies of the atom/vacancy diffusion were calculated using the nudged elastic band (NEB) method $^{29,30}$.
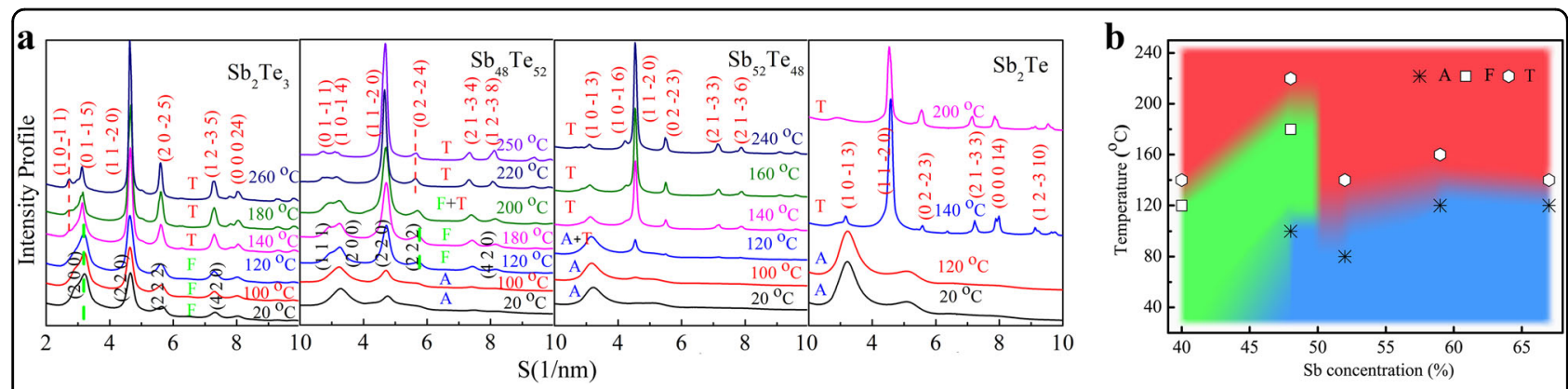

Fig. 1 The electron diffraction profiles showing the structure evolutions of Sb-Te binary compounds in in-situ TEM heating experiments. a Intensity profiles extracted from diffraction rings at an elevated temperature for different $\mathrm{Sb}$ concentrations. $\mathbf{b}$ The temperature of the different phase changes with the variation in the Sb concentration. Blue represents the amorphous phase (A), green represents the FCC phase (F) and red represents the trigonal phase $(T)$ 


\section{Results and discussion}

In situ TEM characterization of the phase transformations of $\mathrm{Sb}$-Te compounds

Figure 1a shows an intensity profile extracted from the diffraction patterns (Supplementary Figs 1-4) of in situ heating experiments for $\mathrm{Sb}-\mathrm{Te}$ compounds with different Sb concentrations. In Fig. 1a, the intensity profiles of the diffraction patterns indicate that partial $\mathrm{Sb}_{2} \mathrm{Te}_{3}$ films crystallized even at room temperature. Some crystalline grains (below $10 \mathrm{~nm}$ ) were found in the TEM bright-field (BF) image (Supplementary Fig. 1). The indexed diffraction pattern shows that the structure at room temperature belonged to the FCC phase. With increasing temperature, an increasing number of large grains $(\sim 10 \mathrm{~nm})$ appeared. The intensity profiles of the diffraction patterns indicated that up to $120^{\circ} \mathrm{C}$, the crystals retained the cubic structure. At $140{ }^{\circ} \mathrm{C}$, the $2 \Theta$ value of the (200) FCC peak decreased, indicating that the phase changed to a trigonal structure.

When the composition approached $\mathrm{SbTe}\left(\mathrm{Sb}_{48} \mathrm{Te}_{52}\right)$, the intensity profile of the as-deposited film was characterized as an amorphous phase. Although some grainlike particles appeared in the $\mathrm{BF}$ image at $100^{\circ} \mathrm{C}$ (Supplementary Fig. 2), the cubic phase did not appear in the intensity profile until $120^{\circ} \mathrm{C}$ and remained until $200^{\circ} \mathrm{C}$. At $200^{\circ} \mathrm{C}$, the (222) peak of the cubic phase shifted slightly to the left, indicating incubation of the trigonal

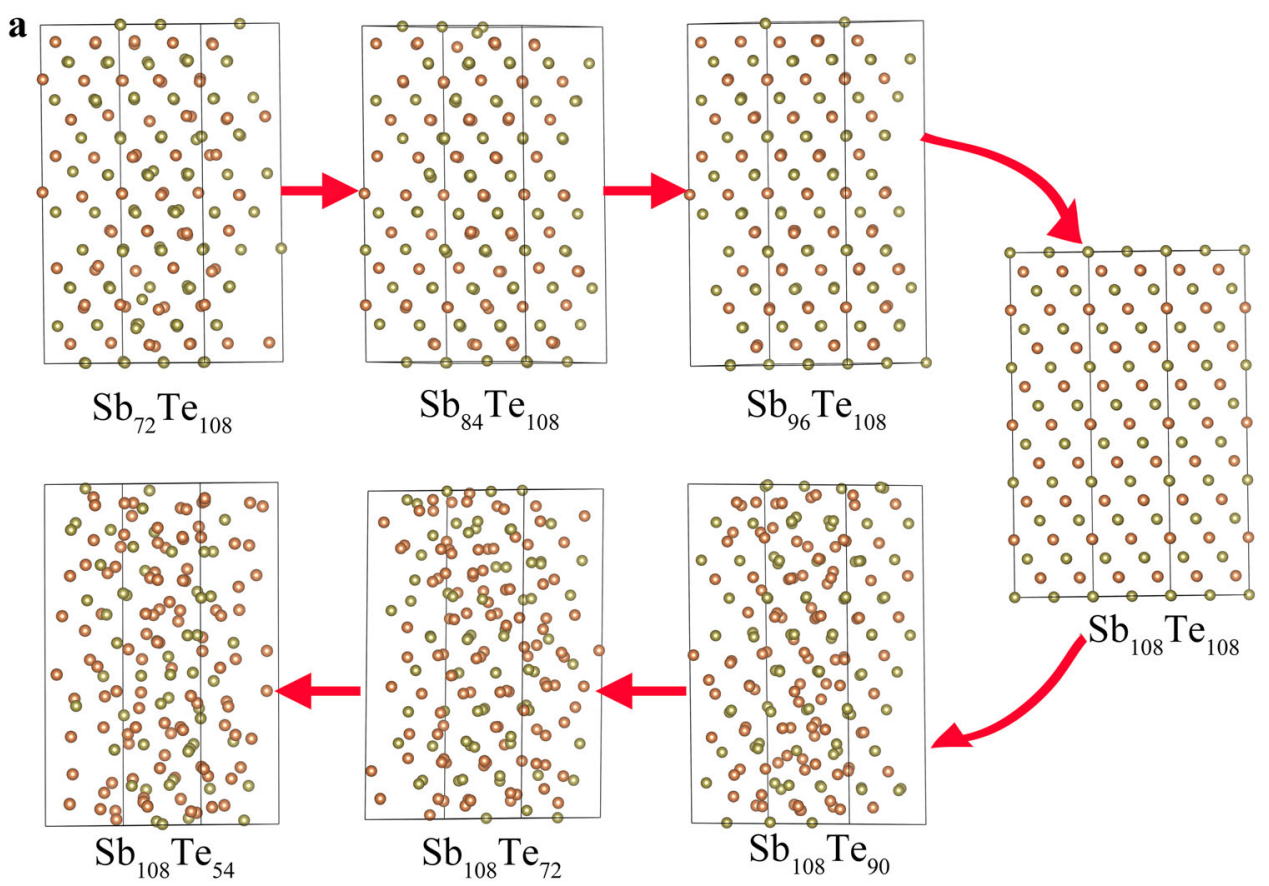

b

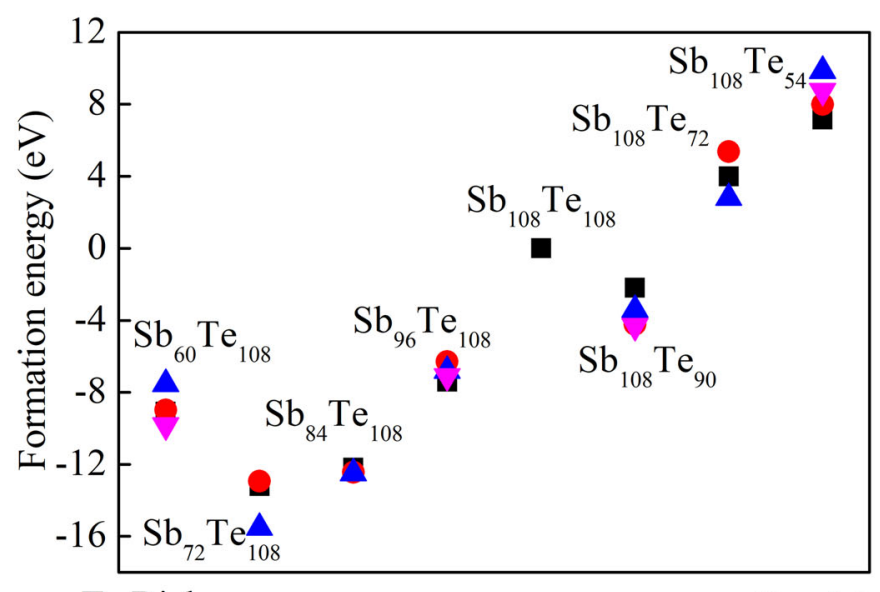

Te Rich

Sb Rich

Fig. 2 FCC models with different types and amounts of vacancies. a Relaxed structures. $\mathbf{b}$ The corresponding formation energies ( $E_{f}$ ) 
phase. When the temperature increased to $220^{\circ} \mathrm{C}$, the intensity profile showed a well-characterized trigonal phase. To confirm the structure of the $\mathrm{Sb}_{48} \mathrm{Te}_{52}$, HRTEM was performed to observe the image of the film after heating to $160^{\circ} \mathrm{C}$ (Supplementary Fig. 5). The HRTEM images of the $<001>,<111>$ and $<110>$ zone axes clearly indicate the existence of the FCC phase, which is quite similar to cubic $\mathrm{Sb}_{2} \mathrm{Te}_{3}{ }^{8}$. However, when the composition crossed the $\mathrm{SbTe}$ line, the phase transition of the compound abruptly changed. As shown in Fig. 1a, the intensity profile indicates that the cubic phase in the above compounds disappeared in the $\mathrm{Sb}_{52} \mathrm{Te}_{48}$ compound. Partial $\mathrm{Sb}_{52} \mathrm{Te}_{48}$ began to directly crystallize to the trigonal phase at $120^{\circ} \mathrm{C}$ (Supplementary Fig. 3). With increasing temperature, additional crystalline diffraction spots appeared. Up to $160^{\circ} \mathrm{C}$, the compound completely crystallized to the trigonal phase. In comparison, when the composition of the $\mathrm{Sb}-\mathrm{Te}$ compound approached $\mathrm{Sb}_{2} \mathrm{Te}$, all the compounds quickly crystallized into the trigonal phase at $140{ }^{\circ} \mathrm{C}$ (Supplementary Fig. 4).

According to the results of the in situ heating experiments, the phase transition temperatures vs various $\mathrm{Sb}$ concentrations are summarized in Fig. 1b. As shown in the figure, a distinct boundary appeared when the $\mathrm{Sb}$ concentration equaled $50 \%$. When the $\mathrm{Sb}$ concentration was greater than $50 \%$, the cubic phase was not observed. The cubic phase was only obtained when the Sb concentration was lower than $50 \%$. In addition, the data reveal that the phase transition temperatures of both the amorphous-cubic and cubic-trigonal phases increased with increasing $\mathrm{Sb}$ concentration or decreasing amount of vacancies of the $\mathrm{Sb}$ sites in the cubic $\mathrm{Sb}-\mathrm{Te}$ compounds.

\section{$\mathrm{Ab}$ initio calculations revealing the stability of FCC Sb-Te structures with different types and amounts of vacancies}

To understand the in situ heating TEM experimental results, we performed ab initio calculations. For a comparison with the trigonal phase, a hexagonal supercell with P1 symmetry was designed according to the FCC structure, with $108 \mathrm{Sb}$ atoms on one sublattice and $108 \mathrm{Te}$ atoms on the other sublattice. Based on this structure, $\mathrm{Sb}$ and Te atoms were removed randomly to create vacancies, and then the structures were relaxed to their ground state energy. By removing $36 \mathrm{Sb}$ atoms, the composition of the supercell was the same as $\mathrm{Sb}_{2} \mathrm{Te}_{3}$. By removing 54 Te atoms, the composition of the supercell was the same as $\mathrm{Sb}_{2} \mathrm{Te}$.

As presented by the atomic models in Fig. 2a, the structural frameworks of the cubic phase remained well defined after removing the $\mathrm{Sb}$ atoms, even when the number of atoms removed reached 36. In contrast, the removal of $18 \mathrm{Te}$ atoms severely deformed the local structures. After removing $36 \mathrm{Te}$ atoms, the framework of the cubic structure was completely destroyed, and the situation deteriorated after removing $54 \mathrm{Te}$ atoms. In addition, the corresponding formation energy $\left(E_{f}\right)$ was also calculated to reveal the stability of the cubic structures.

As depicted in Fig. 2b, the cubic structure with $\mathrm{Sb}$ vacancies had a negative $E_{f}$. The value of $E_{f}$ was the lowest
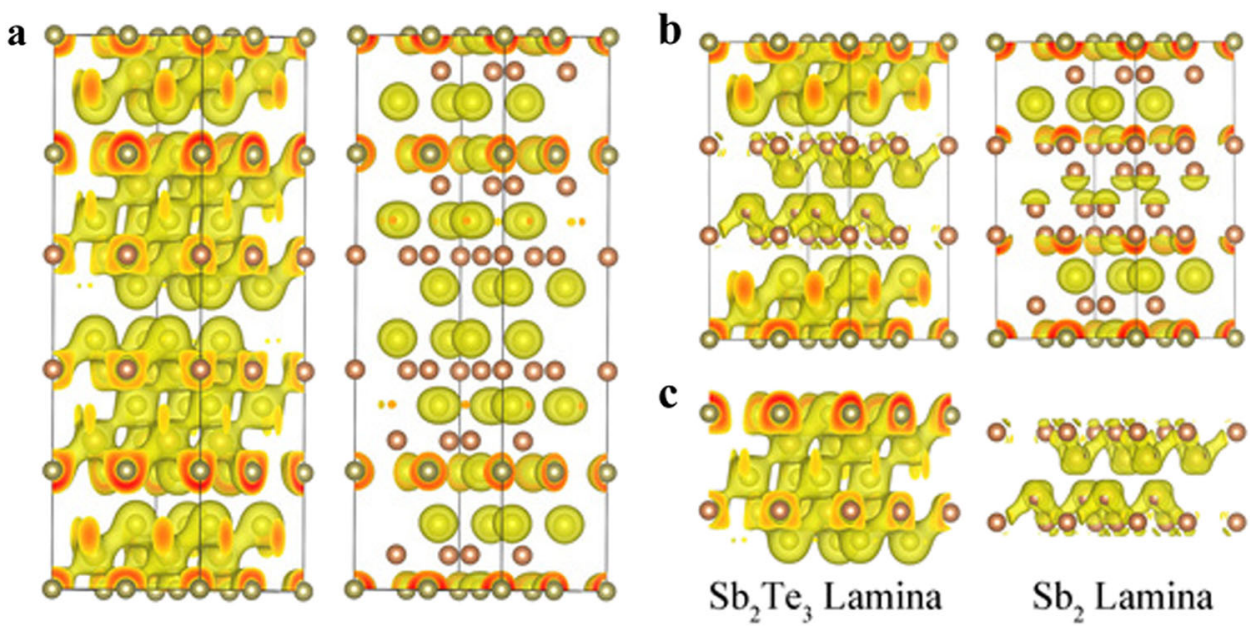

Fig. 3 The charge denstiy maps showing the bonding and antibonding states of stable trigonal $\mathrm{Sb}_{2} \mathrm{Te}_{3}$ and $\mathrm{Sb}_{2} \mathrm{Te}$ phases. a, $\mathbf{b} C h a r g e$ density maps of the bonding states and antibonding states for trigonal $\mathrm{Sb}_{2} \mathrm{Te}_{3}$ and $\mathrm{Sb}_{2} \mathrm{Te} . \mathbf{c} \mathrm{Sb}_{2} \mathrm{Te}_{3}$ and $\mathrm{Sb}_{2}$ lamina extracted from the $\mathrm{Sb}_{2} \mathrm{Te} e_{3}$ and $\mathrm{Sb}_{2} \mathrm{Te}$ trigonal phases. The bonding and antibonding energy ranges were selected from the COHPs in Supplementary Fig. 6. For trigonal $\mathrm{Sb}_{2} \mathrm{Te}_{3}$, the energy window for the charge density map of the antibonding states was $-1.95 \sim 0 \mathrm{eV}$, and the energy window of the bonding states was -5.87 to approximately $-2.00 \mathrm{eV}$. For trigonal $\mathrm{Sb}_{2} \mathrm{Te}$, the corresponding energy windows were -2.30 to $\sim 0 \mathrm{eV}$ and -6.07 to $\sim-2.36 \mathrm{eV}$, respectively. The isosurfaces for the antibonding states were set to $60 \%$ of the maximum point, and those for the bonding states were set to $50 \%$ of the maximum point 


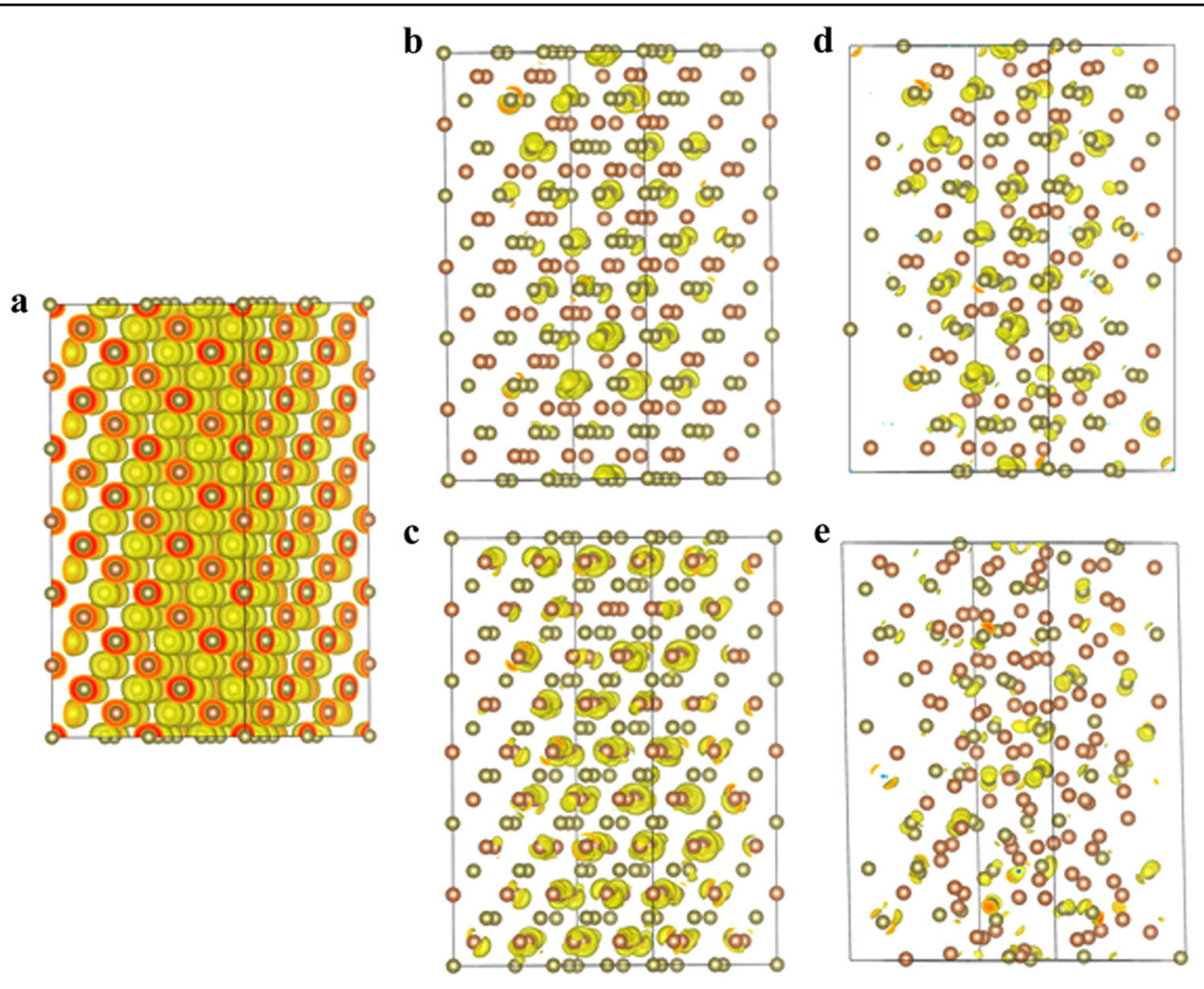

Fig. 4 Charge density maps of the antibonding states for the theoretical FCC structure of a Sb${ }_{108} \mathrm{Te}_{108}, \mathrm{~b} \mathrm{Sb}_{72} \mathrm{Te}_{108}$ and c Sb $\mathrm{b}_{108} \mathrm{Te}_{72}$ and the corresponding relaxed structures of $\mathbf{d} \mathbf{S b}_{\mathbf{7 2}} \mathrm{Te}_{108}$ and $\mathbf{e} \mathbf{S b}_{108} \mathrm{Te}_{\mathbf{7 2}}$. The antibonding energy ranges were selected from the COHP in Supplementary Fig. 7. The energy window for $\mathrm{Sb}_{108} \mathrm{Te}_{108}$ was $-2.5 \sim 0 \mathrm{eV}$. The energy window for both the relaxed and unrelaxed structures of $\mathrm{Sb}_{72} \mathrm{Te}_{108}$ was $-1.9 \sim 0 \mathrm{eV}$. The energy windows for the relaxed and unrelaxed structures of $\mathrm{Sb}_{108} \mathrm{Te}_{72}$ were $-1.8 \sim 0 \mathrm{eV}$ and $-2.4 \sim 0 \mathrm{eV}$, respectively. The iso-surfaces are set to $60 \%$ of the maximum point

when the composition approached $\mathrm{Sb}_{72} \mathrm{Te}_{108}$ (namely, $\mathrm{Sb}_{2} \mathrm{Te}_{3}$ ), and $\mathrm{E}_{\mathrm{f}}$ gradually increased to zero as the $\mathrm{Sb}$ concentration approached $50 \%$. The low value of $\mathrm{E}_{\mathrm{f}}$ means that $\mathrm{Sb}_{2} \mathrm{Te}_{3}$ was more energetically favorable. This explains why the as-deposited $\mathrm{Sb}_{2} \mathrm{Te}_{3}$ crystallized at room temperature. When the $\mathrm{Sb}$ concentration was above 50\%, $\mathrm{E}_{\mathrm{f}}$ increased above zero except for in $\mathrm{Sb}_{108} \mathrm{Te}_{90}$. Even with a negative formation energy, the cubic structure was impossible because of the severe deformation of the structural framework, as shown in Fig. 2a.

\section{Charge density maps revealing the electronic structures of the Sb-Te phase}

To understand what leads to the distinct behaviors of the phase transition for $\mathrm{Sb}-\mathrm{Te}$ compounds, we revisit the basic electronic structure of $\mathrm{Sb}$ and $\mathrm{Te}$ atoms, which have $\mathrm{s}^{2} \mathrm{p}^{3}$ and $\mathrm{s}^{2} \mathrm{p}^{4}$ outer shell structures, respectively. Therefore, the most favorable bonding environments for $\mathrm{Sb}$ and Te are 3 and 2 p-bonding coordinated atoms, respectively. Figure $3 \mathrm{a}$ and $\mathrm{b}$ shows the charge density maps of the stable phases for $\mathrm{Sb}_{2} \mathrm{Te}_{3}$ and $\mathrm{Sb}_{2} \mathrm{Te}$, respectively.
As shown in the left panel in Fig. 3a, the most stable compound for $\mathrm{Sb}-\mathrm{Te}$ alloys was the $\mathrm{Sb}_{2} \mathrm{Te}_{3}$ trigonal phase, where, on average, $\mathrm{Sb}$ has 3 coordinated Te atoms and Te has 2 coordinated $\mathrm{Sb}$ atoms. Even for the stable phase, the local coordination environments for $\mathrm{Sb}$ and $\mathrm{Te}$ were not perfect. Thus, antibonding states still existed in the stable phase (Supplementary Fig. 6, the crystal orbital Hamilton populations (COHPs) of $\mathrm{Sb}_{2} \mathrm{Te}_{3}$ and $\mathrm{Sb}_{2} \mathrm{Te}$ ). As shown in the right panel in Fig. 3a, these antibonding states were dominated by electrons surrounding Te. Moreover, in the octahedral bonding environment, $\mathrm{Te}-\mathrm{Te}$ bonding was not favorable because the coordination environment was contradictory to the preferable requirement, which is the right angle zigzag chain structure $^{31}$. In contrast, $\mathrm{Sb}-\mathrm{Sb}$ bonding was more energetically preferable than $\mathrm{Te}-\mathrm{Te}$ bonding because the bonds can be saturated with three perpendicular p-bonds, as shown by the $\mathrm{Sb}_{2}$ lamina in Fig. 3c. For the $\mathrm{Sb}_{2} \mathrm{Te}$ compound, the most stable structure was constructed by one laminar $\mathrm{Sb}_{2} \mathrm{Te}_{3}$ layer plus two extra laminar $\mathrm{Sb}_{2}$ layers (Fig. $3 \mathrm{~b}$ ). These basic facts set up the prerequisites that the alloying structures must obey in the phase transitions. 

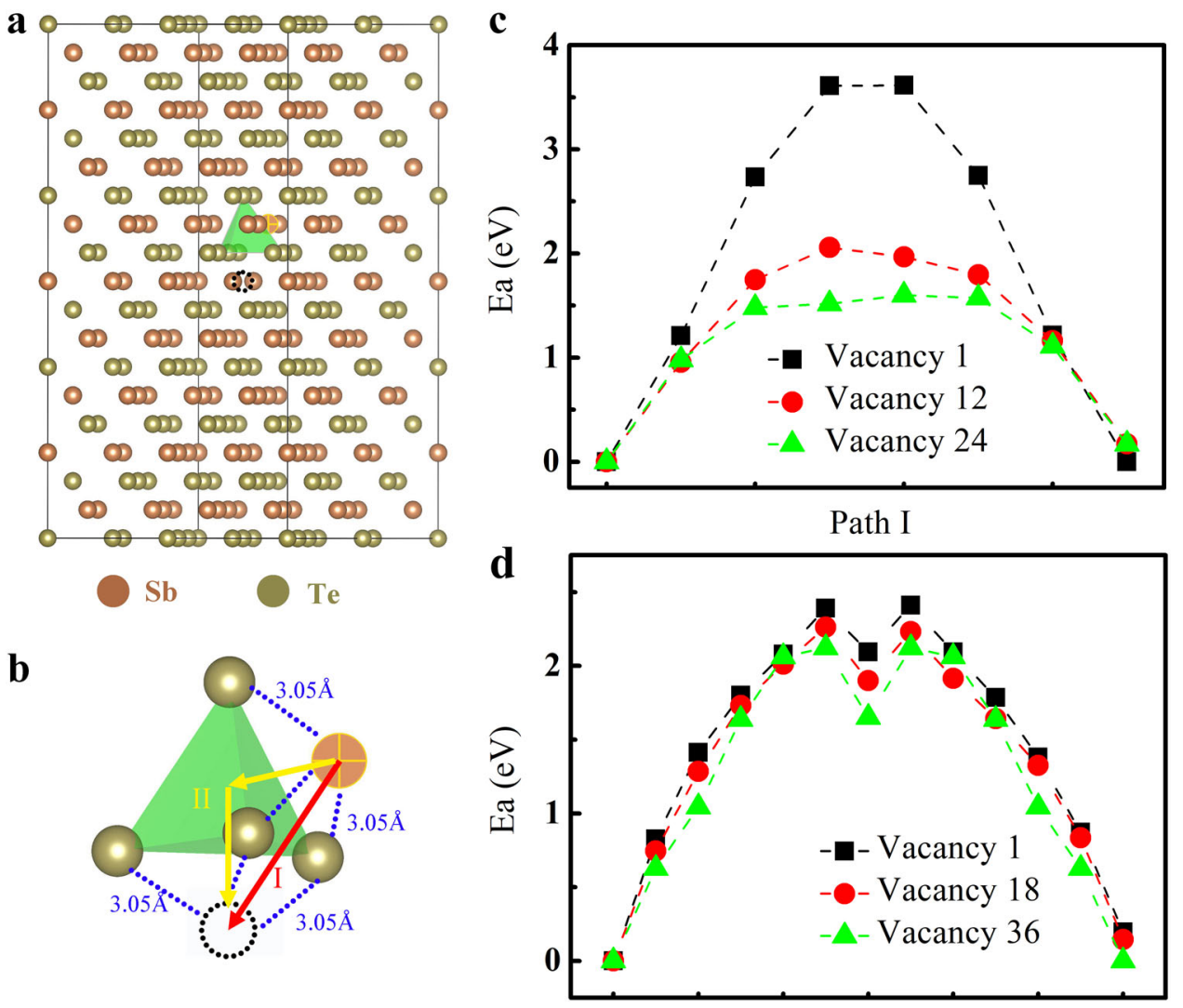

Path II

Fig. 5 The Sb diffusion paths and corresponding energy landscapes in cubic Sb-Te compounds. a Model for Sb atom diffusion, in which the diffusing Sb atom is located near the green tetrahedron, and the black dashed circle indicates the future position of this Sb atom. $\mathbf{b}$ The initial and final positions of the diffusing Sb atom. Two different diffusion paths are indicated by the red line (path I: directly to the final position) and the yellow lines (path II: through the tetrahedral center). $\mathbf{c}$, d The corresponding activation energies $\left(E_{a}\right)$ for path I and path II

When $\mathrm{Sb}$ and Te were arranged into a theoretical rocksalt (RS) structure, there were 7 electrons in the $\mathrm{Sb}$ and $\mathrm{Te}$ p-orbitals. This means that the p-orbitals for $\mathrm{Sb}$ and $\mathrm{Te}$ were overloaded. Thus, the extra electrons in the porbitals were forced into the antibonding states. In this RS structure, as shown in Fig. 4a, the electrons of the antibonding states (Supplementary Fig. 7a) surrounded both $\mathrm{Sb}$ and $\mathrm{Te}$ atoms, and the energy of the $\mathrm{Sb}-\mathrm{Te}$ composite was high. Removing $\mathrm{Sb}$ atoms decreased the formation energy of the cubic $\mathrm{Sb}-\mathrm{Te}$ composites, as shown in Fig. $2 \mathrm{~b}$. In contrast, vacancies of the Te sites greatly increased the formation energy of the $\mathrm{Sb}-\mathrm{Te}$ compounds and destroyed the cubic framework. Figure 4b, c show the charge density maps of the antibonding states for unrelaxed cubic $\mathrm{Sb}_{72} \mathrm{Te}_{108}$ and $\mathrm{Sb}_{108} \mathrm{Te}_{72}$, respectively. As shown in Fig. 4b, after removing $36 \mathrm{Sb}$ atoms, the electrons of the antibonding states were only found around the Te atoms. This is similar to the charge density map of the antibonding states for trigonal $\mathrm{Sb}_{2} \mathrm{Te}_{3}$, indicating a decreased formation energy. However, the vacancies of the Te sites resulted in more antibonding electrons (Supplementary Fig. $7 \mathrm{~b}$ and c). Furthermore, in contrast to $\mathrm{Sb}_{72} \mathrm{Te}_{108}$, the antibonding electrons in $\mathrm{Sb}_{108} \mathrm{Te}_{72}$ mainly surrounded the $\mathrm{Sb}$ atoms, suggesting a relatively high formation energy.

Relaxation further deceased the ground state of a structure by finding more suitable atom positions in a cell. As shown in Fig. 4d, the structure of $\mathrm{Sb}_{72} \mathrm{Te}_{108}$ adjusted slightly due to the similarity of the distributions of the antibonding electrons between cubic and trigonal phases. In contrast, the dramatic difference in the distribution of antibonding electrons in the $\mathrm{Sb}_{108} \mathrm{Te}_{72}$ composite resulted in a high driving force for the relaxation of the structure (this can also be seen from the COHP difference before and after the relaxation in Supplementary Fig. $7 \mathrm{c}$ and d). This difference collapsed the cubic framework. The charge density map in Fig. 4e shows that the atoms surrounded by antibonding electrons changed to Te from $\mathrm{Sb}$ after relaxation. Some $\mathrm{Sb}-\mathrm{Sb}$ bonds appeared after relaxation (Supplementary Fig. 8). These results agree well with the structure of trigonal $\mathrm{Sb}_{2} \mathrm{Te}$. 


\section{Vacancies decreasing the diffusion energy barriers}

The phase transition temperature from the cubic phase to the trigonal phase was determined by the activation energy $\left(E_{a}\right)$ for atom/vacancy diffusion in the cubic framework. The activation of diffusion is required by the vacancy ordering and evaporation in the phase transition. This activation energy is defined by the energy barrier that the atom/vacancy must overcome in the diffusion path. Figure $5 \mathrm{a}$ shows a model for $\mathrm{Sb}$ atom diffusion. The diffusing $\mathrm{Sb}$ atom was situated near the green tetrahedron in Fig. $5 \mathrm{a}$, and the black dashed circle indicates the future position of this $\mathrm{Sb}$ atom. In Fig. 5b, the initial and final positions for this $\mathrm{Sb}$ atom are clearly presented. Two different diffusion paths are also shown, where the red arrow indicates path I (directly to the final position) and the yellow arrow indicates path II (through the tetrahedral center). In the case of decreased vacancies (vacancy 1 ), the activation energy $\left(E_{a}\right)$ required for the $\mathrm{Sb}$ atom to cross the energy barrier in path II was obviously lower than that in path I, as shown by Fig. 5c, d, respectively. With an increase in the number of vacancies, $E_{a}$ shows a sharper decrease for path I than for path II. Although the number of vacancies had a different impact on $E_{a}$ for path I and path II, $E_{a}$ decreased as the number of vacancies increased in both cases. More vacancies means a large space to relax in during the atom diffusion process, which decreased the energy barriers that must be overcome by the diffusing atoms. This agrees well with our experimental results and the corresponding phenomenon in $\mathrm{GeTe}-\mathrm{Sb}_{2} \mathrm{Te}_{3}$ pseudobinary alloys, where more $\mathrm{Sb}_{2} \mathrm{Te}_{3}$ means a decreased phase transition temperature?

The metastable cubic phases in our in situ TEM experiments only appeared when the $\mathrm{Sb}$ concentration was lower than $50 \%$. This special composition-dependent phase transition was also demonstrated in thick samples $(\sim 100 \mathrm{~nm})$ by resistance-temperature (R-T) measurements (Supplementary Fig. 9).

In summary, in situ heating TEM experiments combined with ab initial calculations were employed to understand the phase transitions in Sb-Te binary compounds. Vacancies were found to have a strong impact on the stability of cubic phases for $\mathrm{Sb}-\mathrm{Te}$ compounds. The distribution of antibonding electrons supplied the driving force for the structural transitions. Furthermore, the results also showed that reducing the vacancies greatly increased the phase transition temperatures for both amorphous-cubic and cubic-trigonal phases, thus improving the data retention ability and cyclability. Doping may reduce the vacancies of the crystalline phase. Moreover, this also increased the stability of the amorphous phase ${ }^{9}$, further improving the data retention ability. Doping $\mathrm{Sb}-\mathrm{Te}$ compounds may provide an approach to discover better phase change materials for DRAM applications.

\begin{abstract}
Acknowledgements
This work was financially supported by the National Key Research and Development Program of China (2017YFB0206101) and the National Natural Science Foundation of China (61504157). We would like to thank T. Li for the PCF analysis, X. Y. Wang, Y. Wang and Dr D. Han for useful discussions.
\end{abstract}

\section{Author details}

${ }^{1}$ State Key Laboratory of Functional Materials for Informatics, Shanghai Institute of Micro-system and Information Technology, Chinese Academy of Sciences, 200050 Shanghai, China. ${ }^{2}$ Institute of Microstructure and Property of Advanced Materials, Beijing University of Technology, 100124 Beijing, China. ${ }^{3}$ School of Physical Science and Technology, Shanghai Tech University, 201210 Shanghai, China. ${ }^{4}$ Key Laboratory of Polar Materials and Devices (MOE), Department of Optoelectronics, East China Normal University, 200241 Shanghai, China

\section{Author contributions}

X.C. performed the in situ TEM observation and ab initio calculations. Y.C. designed the in situ heating TEM experiments. X.C. and X.L. analyzed the experimental and theoretical results. X.L. and X.C. wrote the paper with the help from all coauthors. The project was initiated and conceptualized by X.L. and Z.S.

\section{Conflict of interest}

The authors declare that they have no conflict of interest.

\section{Publisher's note}

Springer Nature remains neutral with regard to jurisdictional claims in published maps and institutional affiliations.

Supplementary information is available for this paper at https://doi.org/ 10.1038/s41427-019-0140-z.

Received: 7 February 2019 Accepted: 24 May 2019.

Published online: 26 July 2019

\section{References}

1. Sarwat, S. G. et al. Scaling limits of graphene nanoelectrodes. Nano Lett. 17, 3688-3693 (2017).

2. Sarwat, S. G. Materials science and engineering of phase change random access memory. Mater. Sci. Technol. 33, 1890-1906 (2017).

3. Wong, H. S. P. \& Salahuddin, S. Memory leads the way to better computing. Nat. Nanotechnol. 10, 191 (2015).

4. Raoux, S., Xiong, F., Wuttig, M. \& Pop, E. Phase change materials and phase change memory. MRS Bull. 39, 703-710 (2014).

5. Burr, G. W. et al. Phase change memory technology. J. Vac. Sci. Technol. 28, 223-262 (2010)

6. Wuttig, M. \& Yamada, N. Phase-change materials for rewriteable data storage. Nat. Mater. 6, 824-832 (2007).

7. Rao, F. et al. Reducing the stochasticity of crystal nucleation to enable subnanosecond memory writing. Science 1427, 1-9 (2017).

8. Zheng, Y. et al. Direct observation of metastable face-centered cubic $\mathrm{Sb}_{2} \mathrm{Te}_{3}$ crystal. Nano Res. 9, 3453-3462 (2016).

9. Wuttig, M. et al. The role of vacancies and local distortions in the design of new phase-change materials. Nat. Mater. 6, 122-128 (2007).

10. Liu, X. Q. et al. New structural picture of the $\mathrm{Ge}_{2} \mathrm{Sb}_{2} \mathrm{Te}_{5}$ phase-change alloy. Phys. Rev. Lett. 106, 025501 (2011).

11. Kolobov, A. V., Fons, P., Tominaga, J. \& Ovshinsky, S. R. Vacancy-mediated three-center four-electron bonds in GeTe-Sb2Te3 phase-change memory alloys. Phys. Rev. B: Condens. Matter Mater. Phys. 87, 1-9 (2013).

12. Siegrist, $\mathrm{T}$. et al. Disorder-induced localization in crystalline phase-change materials. Nat. mater. 10, 202-208 (2011).

13. Zhang, W. et al. Role of vacancies in metal-insulator transitions of crystalline phase-change materials. Nat. Mater. 11, 952-956 (2012).

14. Bragaglia, V. et al. Metal - Insulator Transition Driven by Vacancy Ordering in GeSbTe Phase Change Materials. Sci. Rep. 6, 23843 (2016).

15. Siegert, K. S. et al. Impact of vacancy ordering on thermal transport in crystalline phase-change materials. Rep. Prog. Phys. 78, 013001 (2015). 
16. Behrens, M. et al. Impact of disorder on optical reflectivity contrast of epitaxial $\mathrm{Ge}_{2} \mathrm{Sb}_{2} \mathrm{Te}_{5}$ thin films. Crystengcomm 20, 3688 (2018).

17. Duck, M. M. et al. Disorder control in crystalline $\mathrm{GeSb}_{2} \mathrm{Te}_{4}$ and its impact on characteristic length scales. Rapid Res. Lett. 13, 1800578 (2019).

18. Kim, I. S. et al. High performance PRAM cell scalable to sub-20nm technology with below $4 \mathrm{~F}^{2}$ cell size, extendable to DRAM applications. in Digest of Technical Papers - Symposium on VLSI Technology 203-204 (2010).

19. Zhu, M. et al. One order of magnitude faster phase change at reduced power in Ti-Sb-Te. Nat. Commun. 5, 1-6 (2014)

20. Ding, $K$. et al. The impact of $W$ doping on the phase change behavior of $\mathrm{Sb}_{2} \mathrm{Te}_{3}$. J. Alloy. Compd. 688, 22-26 (2016).

21. Njoroge, W. K. Wöltgens, H.-W. \& Wuttig, M. Density changes upon crystallization of $\mathrm{Ge}_{2} \mathrm{Sb}_{204} \mathrm{Te}_{474}$ films. J. Vac. Sci. Technol. A 20, 230-233 (2002).

22. Kresse, G. \& Furthmüller, J. Efficiency of ab-initio total energy calculations for metals and semiconductors using a plane-wave basis set. Comput. Mater. Sci 6, 15-50 (1996).

23. Joubert, D. From ultrasoft pseudopotentials to the projector augmented-wave method. Phys. Rev. B: Condens. Matter Mater. Phys. 59, 1758 (1999).

24. Perdew, J. P., Burke, K. \& Ernzerhof, M. Generalized gradient approximation made simple. Phys. Rev. Lett. 77, 3865-3868 (1996).
25. Grimme, S. Semiempirical GGA-type density functional constructed with a long-range dispersion correction. J. Comput. Chem. 27, 1787-1799 (2006).

26. Dronskowski, R. \& Blöchl, P. E. Crystal orbital Hamilton populations (COHP) Energy-resolved visualization of chemical bonding in solids based on densityfunctional calculations. J. Phys. Chem. 97, 8617-8624 (1993).

27. Maintz, S., Deringer, V. L., Tchougreeff, A. L. \& Dronskowski, R. LOBSTER: A tool to extract chemical bonding from plane-wave based DFT. J. Comput. Chem. 37, 1030-1035 (2016).

28. Deringer, V. L., Tchougréeff, A. L. \& Dronskowski, R. Crystal orbital Hamilton population (COHP) analysis as projected from plane-wave basis sets. J. Phys. Chem. A 115, 5461-5466 (2011).

29. Henkelman, G., Uberuaga, B. P. \& Jónsson, H. Climbing image nudged elastic band method for finding saddle points and minimum energy paths. J. Chem Phys. 113, 9901-9904 (2000).

30. Henkelman, G. \& Jónsson, H. Improved tangent estimate in the nudged elastic band method for finding minimum energy paths and saddle points. J. Chem. Phys. 113, 9978-9985 (2000)

31. Gaspard, J. P., Pellegatti, A., Marinelli, F. \& Bichara, C. Peierls instabilities in covalent structures I. Electronic structure, cohesion and the $Z=8-\mathrm{N}$ rule Philos. Mag. B 77, 727-744 (1998). 